\title{
Stabilizing a Laminar Flow over a Rectangular Cylinder using Two Small Rotating Cylinders
}

\author{
M. Najafi and M. Goodarzi ${ }^{\dagger}$ \\ Faculty of Engineering, Bu-Ali Sina University, Hamedan, 65174, Iran \\ †Corresponding Author E-mail: m.goodarzi@basu.ac.ir
}

(Received April 19, 2021; accepted September 1, 2021)

\begin{abstract}
The possibility of suppressing laminar vortex shedding after a rectangular cylinder with a pair of rotating controllers has been investigated for $\mathrm{Re}=150$. Drag and lift coefficients of the rectangular cylinder have been measured at different positions of the controller system. Numerical results show that the controller system suppresses the vortex shedding completely when installing at some suitable positions. It was found that the controller system affects the vorticity intensity in the separation bubbles at these suitable positions. The controller system may completely eliminate the separation bubble or may make them remain in attached to the cylinder. Besides, the effectiveness sensitivity of the controller system to its rotation rate and Reynolds number was analyzed when installing at the edge of the cylinder boundary layer where its position is denoted by $\left(r / a=2, \theta=40^{\circ}\right)$. These analyses show that the controller system can still suppress the laminar vortex shedding completely when the rotation rate of the controller system and Reynolds number of the incoming flow change by less than $50 \%$.
\end{abstract}

Keywords: Rectangular cylinder; Rotating controller; Stabilized laminar flow; Laminar vortex shedding; Unstable laminar flow.

\section{INTRODUCTION}

Long cylindrical structures are frequently used in the industry and engineering applications. In some cases, a uniform laminar flow crosses the cylindrical structure. When the inertia of the upcoming steady laminar flow increases, some unstable laminar flows may be generated after the cylinder. This unstable laminar flow induces some vibrations in the cylinder. These vibrations decrease the lifetime of the cylindrical structure. Such an unstable laminar flow concerns with the harmonic vortex shedding to the downstream, beginning from the separated flow behind the cylinder (Fox et al. 2011; White 2010). Vortex shedding causes the oscillatory hydrodynamic forces on the cylinder.

There are different modes of the vortex shedding after a stationary circular cylinder (Williamson 1989). Zdravkovidh (2003), Sumer and Fredsoe (2006), and Tropea et al. (2007) presented complete reviews on the experimental and theoretical studies on the flow over a circular cylinder. Maurel and Petitjeans (1999), and Brocchini and Trivellato (2006) investigated the mechanism of vortex shedding over a circular cylinder comprehensively. Vortex shedding may also be generated after a rotating cylinder. Badr et al. (1989) studied the flow over a rotating cylinder experimentally. Mittal and Kumar (2003) showed that the rotation rate of the cylinder affected the vortex shedding in the wake region.

Shimizu and Tanida (1987), and Sohankar et al. (1998) studied the vortex shedding after a rectangular cylinder. The sharp edges of the rectangular cylinder bring the separation point more upstream. Hence, vortex shedding starts after this particular cylinder even at lower Reynolds number compared to the circular one.

Instability control in the flow after the cylindrical structures is one of the most important subjects in engineering applications. Control methods are classified to active and passive methods. Rashidi et al. (2016) reviewed the different methods for controlling the vortex shedding after a circular cylinder. Passive methods may partially decrease the intensity of the vortex shedding, but cannot suppress it completely (Zdravkovich 1990; Park et al. 2006). A passive method concerns with the presence of a fixed object near the cylinder (Mittal and Raghuvanshi 2001; Dipankar et al. 2006). Chen and Chuan (2013) showed that the presence of a small cylinder near the wake of the main cylinder could remarkably decrease the intensity of the vortex shedding. 
Suction is an active method for controlling the instability in the flow over a cylinder (Shtendel and Seifert 2014). Chen et al. (2013) showed that suction might suppress the vortex shedding after a circular cylinder completely. Sohankar et al. (2015) used suction and blowing flow to control the flow and heat transfer over a rectangular cylinder. They showed that suction or blowing could considerably affect the flow instability after the cylinder. Mittal (2001) used a small rotating cylinder adjacent to the main cylinder to inject the momentum into the separation bubble. He showed that the rotating controller considerably decreased the instability within the flow after the cylinder. Pralits et al. (2010) investigated the instability, sensitivity, and different modes of the vortex shedding after a circular cylinder in the presence of a rotating cylinder.

Jian Sheng et al. (2013) numerically studied the hydrodynamic of the flow over a circular cylinder, using two rotating controllers. Muddada and Patnaik (2010) experimentally showed that two rotating controllers could decrease the vortex shedding in a laminar flow over a circular cylinder. Note that the positions of the rotating controllers were fixed and symmetric in all of the above researches. Goodarzi and Khalili Dehkordi (2017) changed the radial and angular positions of the pair of rotating controllers gradually to find the particular position providing the complete vortex shedding suppression. Khalili Dehkordi et al. (2018) found the particular stable case corresponding to the better hydrodynamic performance.

Goodarzi and Khalili Dehkordi (2017), and Khalili Dehkordi et al. (2018) neither explained how the pair of rotating controllers could suppress the laminar harmonic vortex shedding, nor analyzed the sensitivity of the suppressing mechanism to the changes of the Reynolds number and rotation rate. Besides, it remains to examine the effectiveness of the rotating controller system for other cylindrical structures, especially speaking, rectangular one. In the present study, the same pair of rotating controllers is used to find the possible position of the controller system providing the complete vortex shedding suppression after a rectangular cylinder. Besides, the details of the flow field are investigated to show how the pair of rotating controllers suppresses the vortex shedding. Also, a sensitivity analysis is performed to examine the marginality of the control system effectiveness.

\section{PROBLEM DESCRIPTION}

An incompressible laminar flow is approaching to a rectangular cylinder with square cross section. Two small rotating controllers are symmetrically allocated near the cylinder. Two controllers are rotating symmetrically with respect to the flow direction. The upper controller rotates clockwise. The position of the top rotating controller is presented with the radial distance from the cylinder center and also angular measurement from the geometrical centerline. Figure 1 shows the flow field schematically.
The flow regime is characterized by computing Reynolds number based on the inflow velocity, $U$, and face length of the rectangular cylinder, $a$

$$
\operatorname{Re}=\frac{\rho U a}{\mu}
$$

Reynolds number is 150 as was frequently studied in the literature (Mittal 2001; Goodarzi and Khalili Dehkordi 2017). The ratio of the rotating controller diameter to the rectangular cylinder face-length, i.e., $d / a$, is 0.2 (Mittal 2001; Goodarzi and Khalili Dehkordi 2017). This particular flow regime is corresponding to the laminar harmonic vortex shedding after the cylinder (Zdravkovich 1990). The dimensionless rotation rate of the controller is defined as

$\alpha=\frac{\omega d}{2 U}$

where, $\omega$ is the angular velocity of the rotating controller. The dimensionless rotation rate is 1.2 as was studied by Goodarzi and Khalili Dehkordi (2017)

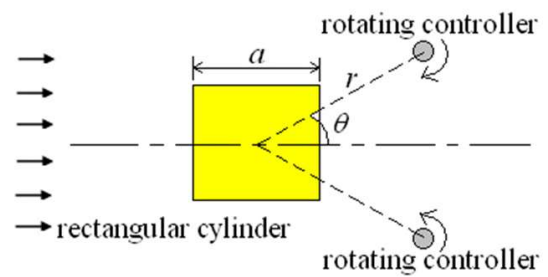

Fig. 1. Schematic presentation of the flow field.

\section{MATHEMATICAL MODEL}

The governing equations for a transient, 2D incompressible, and laminar flow are continuity and momentum equations (Fox et al. 2011). They are presented in a Cartesian coordinate system by the following equations

$$
\begin{aligned}
& \frac{\partial u}{\partial x}+\frac{\partial v}{\partial y}=0 \\
& \rho\left(\frac{\partial u}{\partial t}+\frac{\partial(u u)}{\partial x}+\frac{\partial(v u)}{\partial y}\right)=-\frac{\partial p}{\partial x}+\frac{\partial \tau_{x y}}{\partial y} \\
& \rho\left(\frac{\partial v}{\partial t}+\frac{\partial(u v)}{\partial x}+\frac{\partial(v v)}{\partial y}\right)=-\frac{\partial p}{\partial y}+\frac{\partial \tau_{y x}}{\partial x}
\end{aligned}
$$

where, $u$ and $v$ are velocity components in the $x$ and $y$ directions, respectively. Meanwhile, $\rho, p$, and $\tau$ are density, pressure, and shear stress, respectively.

\section{INITIAL AND BOUNDARY CONDITIONS}

An initial and appropriate boundary conditions are needed to find the temporal distributions of the 


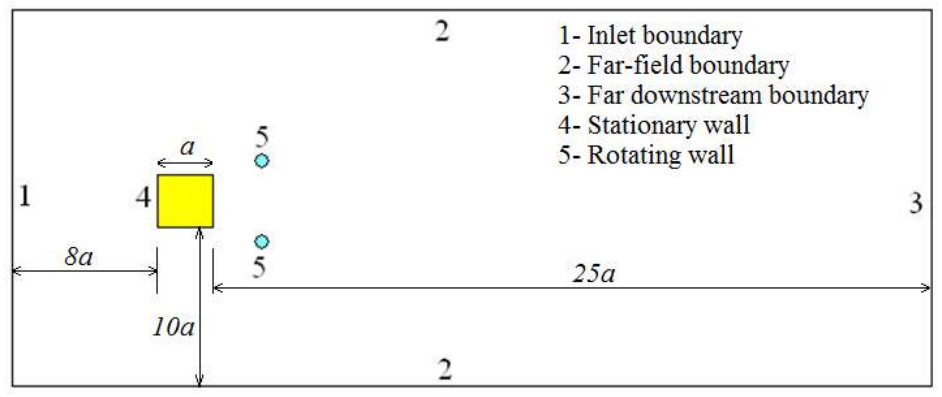

Fig. 2. Definition of the boundary conditions.

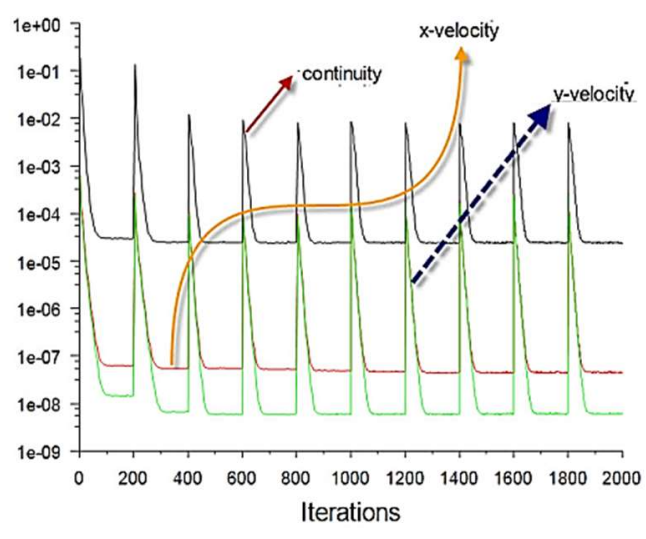

Fig. 3. Typical variations of the computed residuals during the transient simulation.

dependent variables in the flow field. Figure 2 schematically shows the dimensions of the computational flow field and also five different boundary conditions. The upstream flow enters the flow field from the boundary denoted by 1 . A uniform flow is defined at this particular boundary. At the far-field boundary, the variations of all dependent variables are assumed to be vanished, so all gradients of the dependent variables normal to this particular boundary are set to zero. The fardownstream boundary is numerically allocated far enough from the cylinder such that it has negligible effect on the near field. The no-slip condition denotes that the velocity components are zero on the cylinder. It also denotes that the velocity vector on the rotating controller must be equal to its tangential velocity.

\section{DETAILS OF THE NUMERICAL METHOD}

An unstructured grid system with triangular cells was used to discrete the flow field. A finite volume method was used to discrete the governing differential equations. The well-known SIMPLE algorithm (Patankar 1986) was used to provide the coupling between the velocity and pressure fields. A second order upwind scheme was used to discrete the convective fluxes on the cell faces. Iterations per time step were continued until all residuals of the continuity and momentum equations met their invariant values. Figure 3 shows the variations of the residuals for a typical case study. Transient computation was continued until the steady periodic condition was reached.

\section{GRID INDEPENDENCE ANALYSIS}

For flow simulation over a finite body, it is required to determine the numerical far-field boundaries. By definition, the numerical far-field boundary is one has no sensible effect on the near-field flow characteristics. Therefore, several simulations were executed for the particular angular position of the controller, i.e., $\theta=90$, and maximum radial distance between the cylinder and controller with different far-field distances. It was found that the inlet, farfield, and far-downstream boundaries should be at least $6 a, 8 a$, and $22 a$ far from cylinder, respectively. The most important parameters to be investigated in this particular flow problem are drag and lift coefficients. They are defined as

$$
\begin{aligned}
& C_{D}=\frac{2 F_{D}}{\rho U^{2} l} \\
& C_{L}=\frac{2 F_{L}}{\rho U^{2} l}
\end{aligned}
$$

where, $F_{D}$ and $F_{L}$ are drag and lift forces, respectively, and $l$ is the characteristic length of the body. The face length of the cylinder, $a$, and the diameter of the rotating controller, $d$, are used as the characteristic length correspondingly. The results of a numerical simulation should be independent of the grid numbers. The grid independence analysis was done for each case study. Figure 4 shows such a typical analysis. This figure shows the mean values of the drag coefficients of the cylinder and rotating controller, and also the mean value of the lift coefficient of the rotating controller computed with different grid systems. It is obvious that the three mentioned coefficients did not change by increasing the grid numbers greater than some particular one. Figure 5 shows an optimum grid system used for a particular case study.

\section{TIME STEP OPTIMIZATION}

From theoretical viewpoint, the most accurate result would be computed with a time step as small as possible in a transient flow field. But it remarkably increases the time and cost of the computation. 


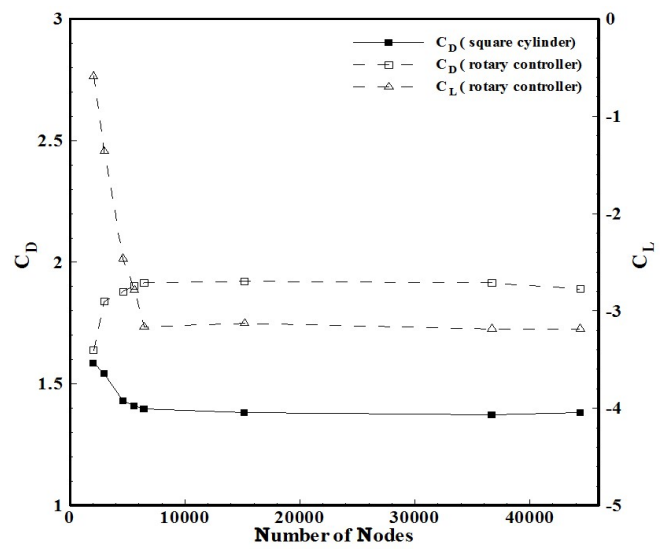

Fig. 4. Grid dependence of the computed drag and lift coefficients of a typical case study.

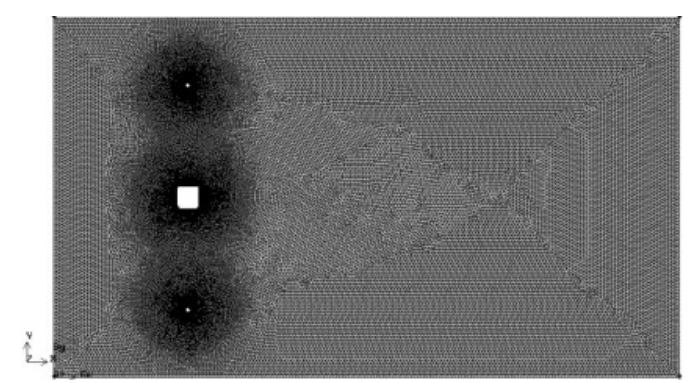

Fig. 5. Grid system used for a particular case study.

Therefore, an optimum time step should be determined. For this purpose, numerical simulation should be executed for a specific time interval with different time steps, beginning with large time step and gradually repeating with smaller ones. Then, the results should be compared to determine the greatest possible time step. Figure 6 shows such an optimum time step determination for a particular case study. This figure shows that the computed results with time steps $0.15,0.1$, and 0.05 are completely the same. So, the numerical simulation could be completely run for this particular case study with $\Delta \mathrm{t}=0.15$ with accurate enough prediction.

\section{VALIDATION}

A numerical method should be validated before applying for a series of simulations. The present numerical method was validated by simulating the flow field over a square cylinder at flow regime $\mathrm{Re}=150$. The dimensionless frequency of the vortex shedding, i.e., Strouhal number, is defined by the following relation

$$
S t=\frac{f a}{U}
$$

where, $f$ is the vortex shedding frequency. Computed results for the mean drag coefficient and Strouhal number are compared in Table 1 with the reported values in the references. There are good agreements between the results of the present numerical method and those have been reported in the references.

\section{RESULTS}

\subsection{Flow patterns over the rectangular cylinder and rotating controllers, $\mathbf{R e}=\mathbf{1 5 0}$}

It is obvious that when the pair of rotating controllers is installed far from the cylinder, it does not affect the flow pattern after the cylinder unless it is within the wake of the cylinder. In the absence of the cylinder, the Reynolds number with respect to the rotating controller diameter is 30 . Literature reported that there was no vortex shedding after a circular cylinder up to $\mathrm{Re}=50$ (Williamson 1989). When the circular cylinder is rotating, this critical Reynolds number increases (Mittal and Kumar 2003). Figure 7 shows the instantaneous vorticity contour plots around the rotating controller installed at $\left(r / a=5, \theta=90^{\circ}\right)$ during a period of the laminar harmonic vortex shedding. Note that there is no vortex shedding after the rotating controller, while the vortexes are shedding after the rectangular cylinder (Fig. 8).

\subsection{Searching for possible complete vortex shedding suppression case, $R e=150$}

In the present study, the location of the controller system is gradually changed both radially and angularly. Since the aim of controlling is to decrease the vortex shedding after the cylinder, the fluctuation of the lift coefficient is very important. The hydrodynamic coefficients of the cylinder in the presence of the controller system are compared to those in the absence of the controller system by introducing the suitable indices. These indices are introduced as the ratio of the former coefficients to the later ones.

Figures 9 and 10 show the variations of the ratios of the root mean squares (RMSs) of the fluctuations of the cylinder lift and drag coefficients. They show that at far distances, i.e., $r / a=5$ and 4 , the controller system cannot decrease the vortex shedding after the cylinder. When the controller system is installed at the far distance within the wake region, i.e., low

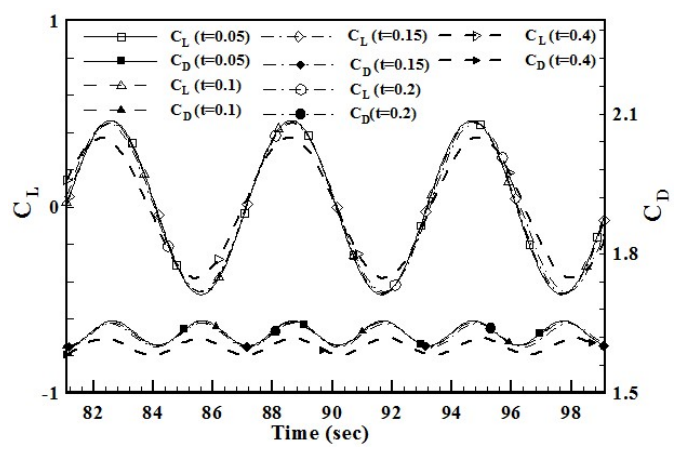

Fig. 6. Comparing obtained results with different time steps for determining the optimum time step. 
M. Najafi and M. Goodarzi/ JAFM, Vol. 15, No. 1, pp. 169-178, 2022

Table 1 Mean drag coefficient and Strouhal number for flow over a square cylinder at $\mathbf{R e}=\mathbf{1 5 0}$

\begin{tabular}{|c|c|c|c|c|c|}
\hline Parameter & $\begin{array}{c}\text { Present } \\
\text { study }\end{array}$ & $\begin{array}{c}\text { Sohankar et al. } \\
(2015)\end{array}$ & $\begin{array}{c}\text { Sohankar } \text { et al. } \\
(2015)\end{array}$ & $\begin{array}{c}\text { Sharma and } \\
\text { Esvaran (2004) }\end{array}$ & $\begin{array}{c}\text { Shimizu and } \\
\text { Tanida (1978) }\end{array}$ \\
\hline$C_{D}$ & 1.467 & 1.440 & 1.440 & 1.465 & 1.491 \\
\hline$S t$ & 0.163 & 0.157 & 0.162 & 0.159 & - \\
\hline
\end{tabular}

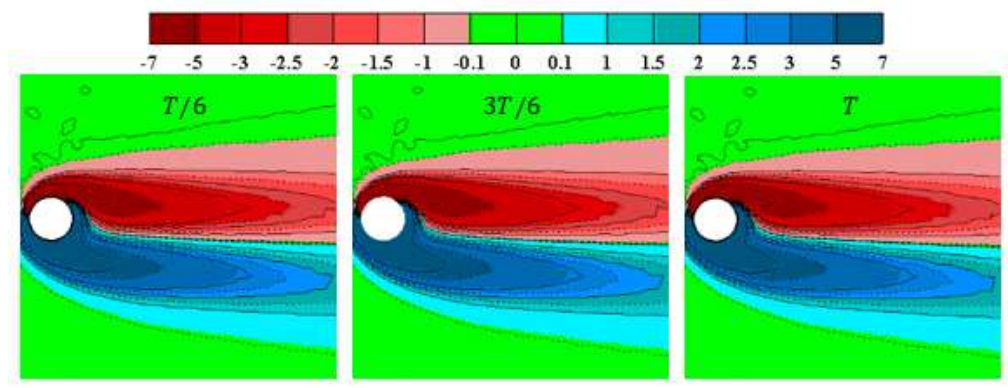

Fig. 7. Instantaneous vorticity contour plots around the rotating controller installed $a t\left(r / a=5, \theta=90^{\circ}\right)$.

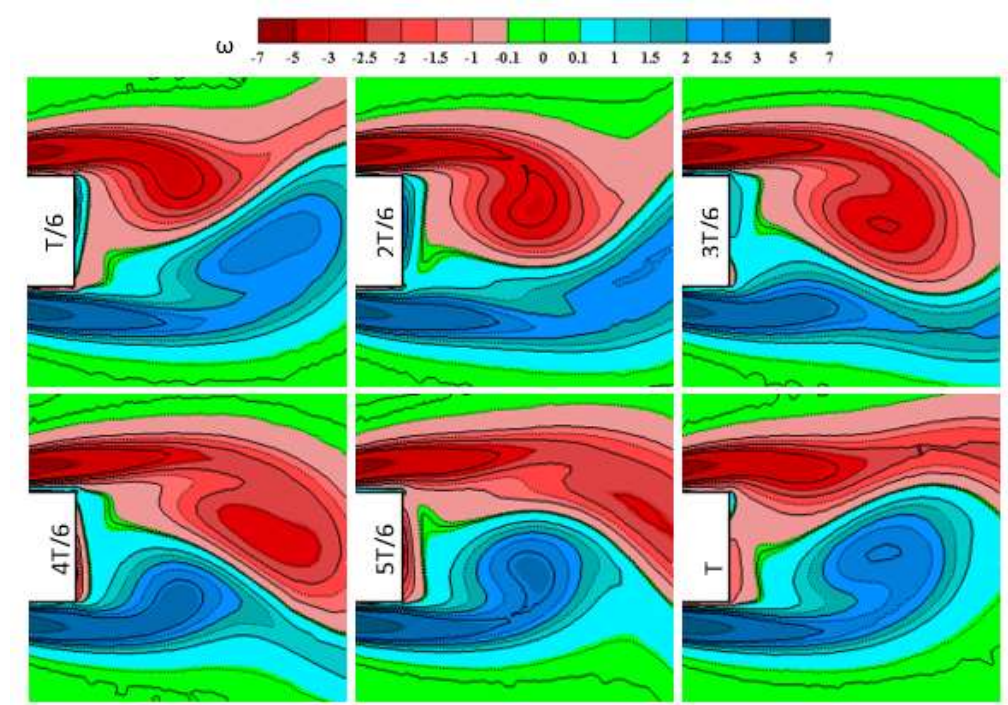

Fig. 8. Vortex shedding after the rectangular cylinder during a period when the rotating controller is installed at $\left(r / a=5, \theta=90^{\circ}\right)$.

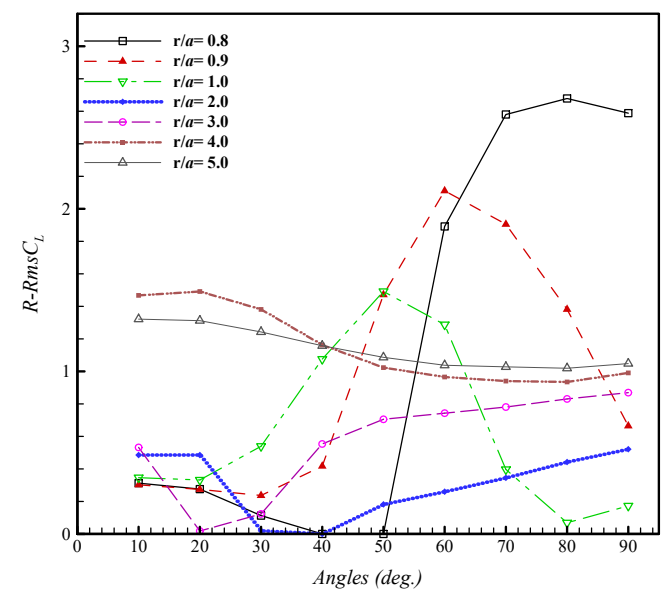

Fig. 9. Ratio of the root mean square of the fluctuations of the cylinder lift coefficient at different positions of the controller system.

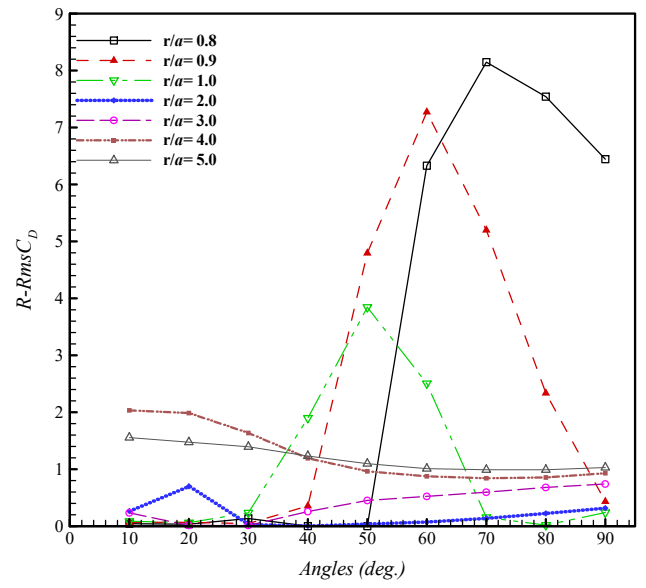

Fig. 10. Ratio of the root mean square of the fluctuation of the cylinder drag coefficient at different positions of the controller system. 
angular position, it increases the cylinder lift fluctuation. Gradually decreasing the radial distance, the favorite effect of the controller system is appeared. When the controller system approaches to the radial distance $r / a=2$, it decreases the lift fluctuation regardless of its angular position. Surprisingly, the fluctuations of the lift and drag coefficients are completely eliminated at the installation position $\left(r / a=2, \theta=40^{\circ}\right)$ for the first time during approaching the controller system to the cylinder. More approaching the controller system to the cylinder shows that the fluctuations of the lift and drag coefficients of the cylinder are completely eliminated at two other positions $\left(\left(r / a=0.8, \theta=50^{\circ}\right)\right.$ and $\left.\left(r / a=0.8, \theta=40^{\circ}\right)\right)$.

Figure 11 shows the streamlines and also vorticity contour plots corresponding to the three mentioned cases which are able to suppress the vortex shedding completely. Note that the thinnest wake among the three cases is established when the controller system is installed at $\left(r / a=0.8, \theta=40^{\circ}\right)$ in which there is no separation bubble behind the cylinder, and even at the downstream.

Table 2 lists the change in the hydrodynamic forces exerting on the cylinder, controller, and the whole of the system at the three mentioned cases. Computed results show that at all cases the drag force on the cylinder and the lift force on the rotating controller decrease compared to those in the absence of the controller system. Of course, the reductions in these forces are not significant when the controller system is installed at $\left(r / a=2, \theta=40^{\circ}\right)$. Note that the drag forces exerting on the rotating controller and the whole of the system increase when the controller system is installed at $\left(r / a=2, \theta=40^{\circ}\right)$. Considering the tabulated results, it seems the controller position $\left(r / a=0.8, \theta=40^{\circ}\right)$ is the best case, but, the controller position $\left(r / a=2, \theta=40^{\circ}\right)$ is safer and more practical than the two other positions, because, there is a little distance between the rotating controller and the main cylinder in the two other cases, which increases the risk of the surface contact and system damage.

\subsection{Description of the mechanism of the complete vortex shedding suppression at $\operatorname{Re}=150$}

In creeping flow, the boundary layer can follow the surface of the cylinder with no separation bubble, because the tangential momentum of the flow within the boundary layer is so low (White 2010). When the Reynolds number of the flow increases to some particular value, the tangential momentum of the flow within the boundary layer increases and the flow does not follow the cylinder surface, because the vorticity intensity increases within the boundary layer. In this case, two separation bubbles in attached to the cylinder with low rotation speeds (low vorticity) are established behind the cylinder (White 2010).

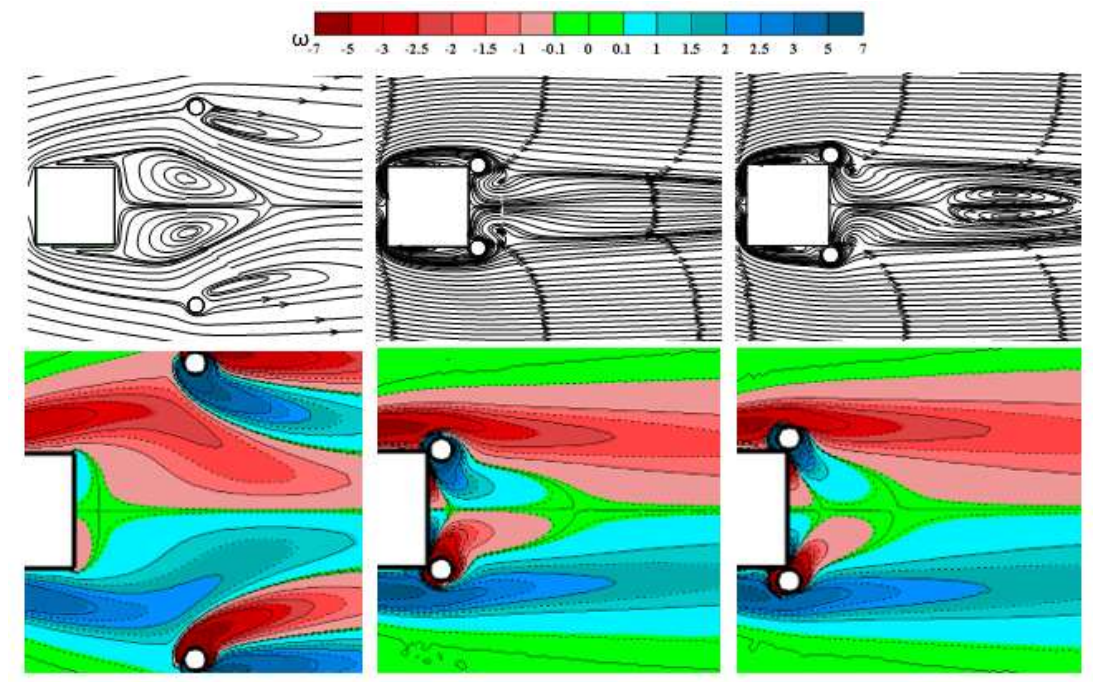

Fig. 11. Streamlines and vorticity contour plots when the vortex shedding is completely suppressed by controller system, left $\left(r / a=2, \theta=40^{\circ}\right)$, middle $\left(r / a=0.8, \theta=40^{\circ}\right)$, and right $\left(r / a=0.8, \theta=50^{\circ}\right)$.

Table 2 Changes in the hydrodynamic forces exerting on the cylinder and controller system at three positions of the controller system corresponding to the complete vortex shedding suppression

\begin{tabular}{|c|c|c|c|}
\hline Controller position & $\left(r / a=2, \theta=40^{\circ}\right)$ & $\left(r / a=0.8, \theta=50^{o}\right)$ & $\left(r / a=0.8, \theta=40^{o}\right)$ \\
\hline$R-C_{D}$ of the cylinder & 0.9 & 0.56 & 0.34 \\
\hline $\mathrm{R}-\mathrm{C}_{\mathrm{D}}$ of the controller & 1.12 & 0.16 & 0.56 \\
\hline $\mathrm{R}-\mathrm{C}_{\mathrm{L}}$ of the controller & 0.96 & 0.24 & 0.30 \\
\hline Reduction percent in the total drag force & $-38 \%$ & $37 \%$ & $42 \%$ \\
\hline
\end{tabular}


Increasing the Reynolds number, the rotation speeds of the separation bubbles are increased. It increases the vortity intensity in the separation bubbles. In this situation, if a noisy vorticity enters the near-wake flow, it may change the vorticity of each separation bubble. The instantaneous difference between the rotation speeds of the two separation bubbles makes the vortex with greater power roll up on the other one and shed to the downstream. This process is changed and repeated harmonically to form a laminar and harmonic Karman Street after the cylinder (Williamson 1989). Therefore, if it is possible to decrease the tangential momentum of the boundary layer and also rotation speeds of the two separation bubbles, it is also possible to suppress the vortex shedding.

In the present flow field, two boundary layers are developed on the cylinder surfaces. Figure 8 shows that there are two small separation bubbles in attached to the upper and lower horizontal surfaces of the cylinder. Two larger separation bubbles harmonically shed after the cylinder in the absence of the controller system (see Fig.8). There is an inner edge between the two developed boundary layers, flapping in the unsteady wake region and joining to the outer edge of each boundary layer during a half period of the vortex shedding. The vorticity intensity at the inner and outer edges of the boundary layers is zero and is declared by the green color in the vorticity contour plots. Figure 12 shows the velocity vector plots in the absence of the controller system and in the presence of the control system when it approaches to the outer edge of the boundary layer at position $\left(r / a=2, \theta=40^{\circ}\right)$. This figure shows that the controller system induces a countercurrent rotation speed to the separation bubble behind the cylinder which decreases the rotation speed and vorticity of the separation bubble. Additionally, the large separation bubbles behind the cylinder are combined with the smaller upstream separation bubbles on the horizontal faces. The presence of the separation bubble after the rotating controller makes the separation bubble of the main cylinder smaller. Now, at this particular case, there are additional edges between the boundary layers developed on the cylinder and rotating controller. They are also declared by the green color in the vorticity contour plots in Fig.13. It seems that additional edges between the developed boundary layers over the main cylinder and rotating controllers increase the region with zero vorticity intensity in the near-wake of the cylinder. Besides, in the presence of the controller system, the separation bubble behind the cylinder is diffused to the upstream attached ones.

Figure 13 shows that when the controller system is allocated at $\left(r / a=0.8, \theta=40^{\circ}\right)$ and $\left(r / a=0.8, \theta=50^{\circ}\right)$, it increases the region of zero vorticity behind the cylinder even more by interfering all edges of the developed boundary layers. The rotating controller occupies the region of the original separation bubble so that the original separation bubble is disappeared. Now, the attached separation bubble on the horizontal face of the cylinder is extended to the downstream. Note that two extended separation bubbles are not in contact to each other anymore in these cases. Hence, they cannot roll up on each other. Considering the mentioned explanations, this theory may be introduced that more interactions between the edges of the developed boundary layers in the presence of the rotating controller system at the appropriate locations may decrease the vorticity intensity in the near-wake region to decrease the instability by vorticity diffusion and eventually to suppress the vortex shedding behind the cylinder.

\subsection{Sensitivity of the controller system to the flow regime}

Sensitivity analyses were performed for suppression effect of the three prescribed controller systems to the somewhat changes in the Reynolds number of the flow. For this purpose, the Reynolds number was changed to 100 and 200, while the rotation rate of the rotating controller is 1.2 .

As was explained in the previous section, the controller system eliminates the separation bubble behind the cylinder when it is at $\left(r / a=0.8, \theta=50^{\circ}, 40^{\circ}\right)$. In these cases, the controller system is within the boundary layers developed on the cylinder. Sensitivity analyses for these controller systems showed that they effectively made the flow steady entirely. Since there is no important point in these particular cases, only the sensitivity analysis of the controller system at $\left(r / a=2, \theta=40^{\circ}\right)$ is investigated. In this particular position the controller system is at the edges of the developed boundary layers on the cylinder. Figure 14 shows the vorticity contour-plots for three different flow regimes when the controller system is at $\left(r / a=2, \theta=40^{\circ}\right)$. Figure 14 shows that the flow pattern within the near-wake of the cylinder is unchanged, but the flow pattern within the wake of the rotating controller changes so that at the Reynolds number 200 the wake of the rotating controller become unsteady. There is a single vortex shedding after each rotating controller at $\mathrm{Re}=200$. So, two parallel unsteady flow patterns are independently established at the far-downstream. Since the controller system is at the edges of the developed

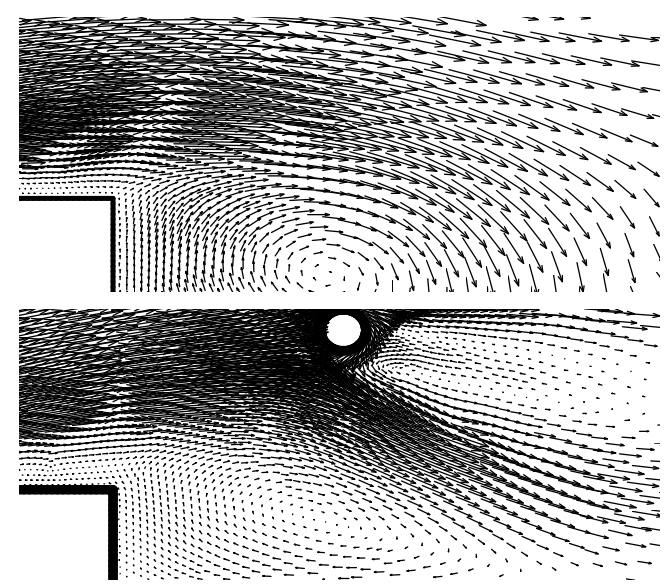

Fig. 12. Comparison between the rotation speeds of the attached separation bubble and shed vortex when the controller system is allocated at $\left(r / a=2, \theta=40^{\circ}\right)$. 


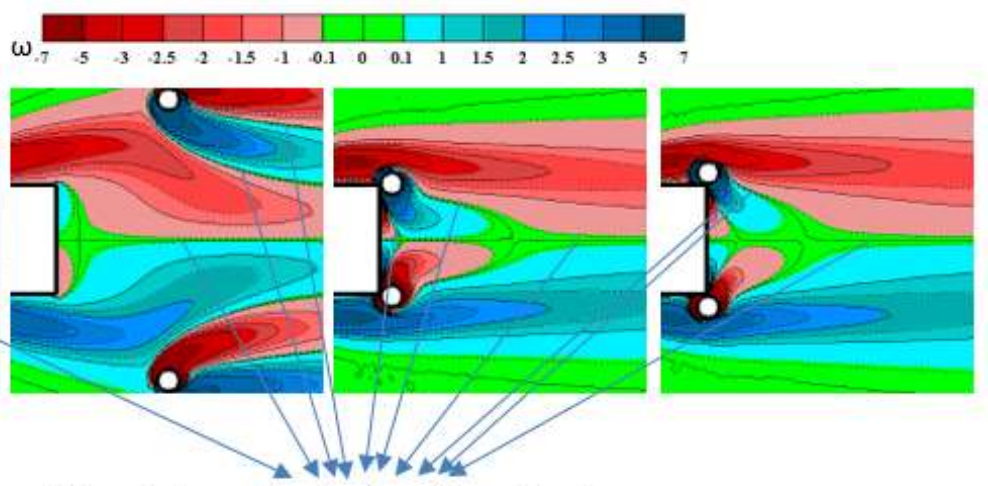

Edges between the developed boundary layers

Fig. 13. Effect of the edges between the developed boundary layers on near-wake region with zero vorticity.
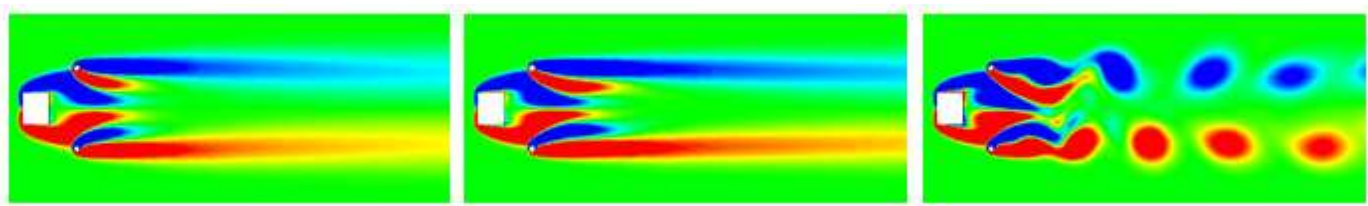

Fig. 14. Instantaneous vorticity contour plots after the system of rectangular and rotating cylinders with $\alpha=1.2$, (left) $\operatorname{Re}=100$, (middle) $R e=150$, and (right) $R e=200$.
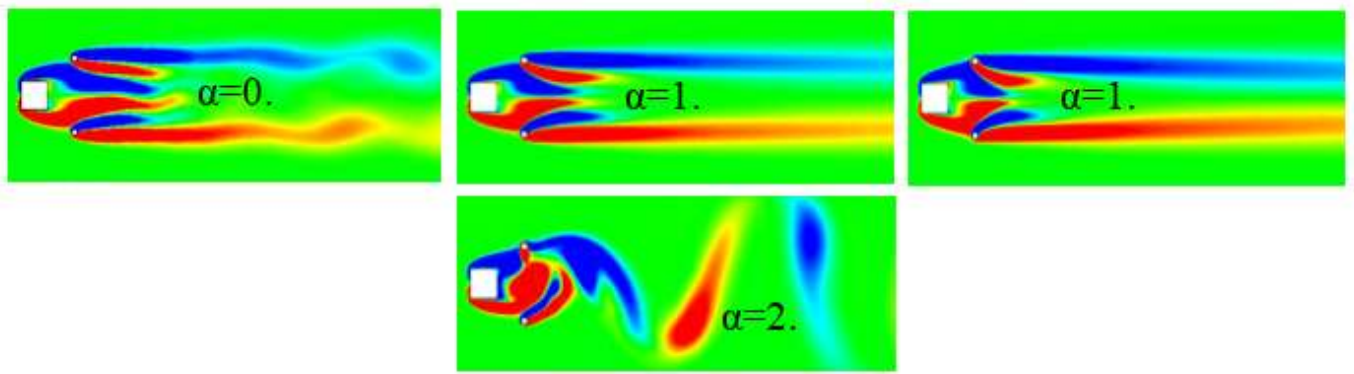

Fig. 15. Instantaneous vorticity contour plots after the system of rectangular and rotating cylinders.

boundary layers on the cylinder, the rotating controllers are partially facing with the flow regime outside the boundary layers. Therefore, they are facing with the higher Reynolds number compared to the cases in which the rotating controllers are installed within the boundary layers. Facing with the flow with higher Reynolds number, makes the flow pattern over the rotating controller unsteady with single vortex shedding, but the flow within the nearwake of the cylinder is still steady.

\subsection{Sensitivity of the controller system to its rotation rate}

Four rotation rates are considered for this sensitivity analysis while the Reynolds number is 150 . Since the suppression effect of the controller system is not affected by the change in the rotation rate of the controller when installing at positions $(r / a=0.8$, $\left.\theta=50^{\circ}, 40^{\circ}\right)$, the sensitivity analysis is performed for installation position $\left(r / a=2, \theta=40^{\circ}\right)$.

Figure 15 shows the vorticity contour plots for different rotation rates when the controller system is installed at $\left(r / a=2, \theta=40^{\circ}\right)$. Except for the rotation rate of $\alpha=2.4$, all hydrodynamic coefficients of the cylinder and rotating controllers are not fluctuating. It means the controller system is able to provide steady hydrodynamics in the near-wakes of the cylinder and rotating controllers. Of course, at rotation rate, $\alpha=0.6$, the separation bubbles are more extended to the downstream so that some weak vortex shedding is happened at the far downstream shear layers of the cylinder and controllers without affecting the near-wakes hydrodynamics. Increasing the rotation rate to $\alpha=1.8$, the separation bubbles are more compressed to the near-wake and become smaller. The flow field is completely steady and the controller system suppresses the vortex shedding completely. Having increased the rotation rate more, $\alpha=2.4$, the separation bubbles behind the rotating controller is shifted to the near-wake of the cylinder and compresses the separation bubble of the cylinder more. Eventually, the separation bubble of the rotating controller rolls up on the rotating controller so that a rotating wake is stablished on the rotating 
controller to produce an unsteady flow pattern on the whole of the system. There is a complicated interaction between the rotating wakes of the rotating controllers and unsteady wake of the cylinder. These wakes are combined together and form a wide Karman Street at the downstream of the whole system. Therefore, the rotation rate of the controller system should not exceed significantly for the controller system at this particular position to have the suitable suppression effectiveness

\section{CONCLUSIONS}

Unstable laminar flow over a rectangular cylinder in the presence of a pair of rotating controllers was investigated numerically. At first, a point by point searching process was conducted to find the possible position of the controller system for complete vortex shedding suppression at the particular flow regime and rotation rate of the controller. Then, the mechanism of the vortex shedding suppression was physically discussed. The sensitivities of the obtained positions to the flow regime and rotation rate of the controller were also investigated. The important conclusions can be addressed as follows.

There are positions for controller system at the flow regime $(\operatorname{Re}=150)$ and controller rotation rate $(\alpha=1.2)$, which suppress the vortex shedding completely. Their radial and angular positions are denoted by $\left(r / a=2, \theta=40^{\circ}\right),\left(r / a=0.8, \theta=40^{\circ}\right)$, and $\left(r / a=0.8, \theta=50^{\circ}\right)$. All of these particular positions for installing the controller system decrease the drag forces exerted on the main cylinder, but the total drag force exerted on the whole of the system including the cylinder and rotating controllers increases somewhat at installing position $\left(r / a=2, \theta=40^{\circ}\right)$. Contrary, when the controller system is installed at $r / a=0.8$, the possibility of surface contacting and structure damaging should be considered in design process. Therefore, the preferred position for installing the controller system depends on the design considerations.

All of the prescribed positions corresponding to the complete vortex shedding increase the region of the zero vorticity in the near-wake of the cylinder by interactions of the edges between the developed boundary layers over the cylinder and rotating controllers. Rotation of the controller induces a negative tangential velocity to the rotating separation bubble after the cylinder when controller system is installed at $\left(r / a=2, \theta=40^{\circ}\right)$. It reduces the rotation speed of the separation bubble and makes the flow regime steady.

The rotating controller system is able to vanish the lift and drag fluctuations on the cylinder even at somewhat higher and less Reynolds numbers. At higher Reynolds number there is a secondary vortex shedding after the rotating controller in the case $\left(r / a=2, \theta=40^{\circ}\right)$, which does not affect the stable flow pattern within the near-wake of the cylinder. The secondary vortex shedding is due to the unsteady flow after the rotating controllers.
Except for significant changes in the rotation rate at case $\left(r / a=2, \theta=40^{\circ}\right)$, the suppression effectiveness of the founded position of the controller system is less sensitive to the rotation rate of the controller.

According to the results of the present study, the position of the controller system, i.e., $r / a=2$ and $\theta=40^{\circ}$, can be successfully and safely used for making the flow over the rectangular cylinder steady in the limited bands of Reynolds number and rotation rate of the controller.

\section{REFERENCES}

Badr, H. M., S. C. R. Dennis and P. J. S. Young (1989). Steady and unsteady flow past a rotating circular cylinder at low Reynolds numbers. Computers and Fluids 17(4), 579609.

Brocchini, M. and F. Trivellato (2006). Vorticity and turbulence effects in fluid structure interaction, an application to hydraulic structure design, WIT Press, ISBN:1-84564-052-7.

Chen, W., H. Hu and H. Li (2013). Suppression of vortex shedding from a circular cylinder by using a suction flow control method, AIAA, 0103 .

Chen, J. C. and P. S. Chuan (2013). Suppression of vortex shedding from a rectangular cylinder at low Reynolds numbers. Journal of Fluids and Structures 43, 15-27.

Dipankar, A., T. K. Sengupta and S. B. Talla (2006). Suppression of vortex shedding behind a circular cylinder by another control cylinder at low Reynolds numbers. Journal of Fluid Mechanics, 1-20.

Fox, M. D., P. J. Pritchard and J. C. Leylegian (2011). Introduction to fluid mechanics, 8th ed., John Wiley \& Sons Inc., ISBN: 0470547553 .

Goodarzi, M. and E. Khalili Dehkordi (2017). Geometrical parameter analysis on stabilizing the flow regime over a circular cylinder using two small rotating controllers. Computers and Fluids 145, 129-140.

Khalili Dehkordi, E., M. Goodarzi and S. H. Nourbakhsh (2018). Optimal active control of laminar flow over a circular cylinder using Taguchi and ANN. European Journal of Mechanics B/Fluids 67, 104-115.

Maurel, A. and P. Petitjeans (1999). Vortex structure and dynamics. lectures of a workshop held in Rouen, Springer, France.

Mittal, S. (2001). Control of flow past bluff bodies using rotating control cylinders. Journal of Fluids and Structures 15, 291-326.

Mittal, S. and A. Raghuvanshi (2001). Control of vortex shedding behind circular cylinder for flows at low Reynolds umbers. International Journal of Numerical Methods in Fluids 35, 421-447. 
Mittal, S. and B. Kumar (2003). Flow past a rotating cylinder. Journal of Fluid Mechanics 476, 303-334.

Muddada, S. and B. S. V. Patnaik (2010). An active flow control strategy for the suppression of vortex structures behind a circular cylinder . European Journal of Mechanics B/Fluids 29, 93-104.

Park, H., D. Lee, W. P. Jeon, S. Hahn and J. Kim (2006). Drag reduction in flow over a twodimensional bluff body with a blunt trailing edge using a new passive device. Journal of Fluid Mechanics 563, 389-414.

Patankar, S. (1980). Numerical heat transfer and fluid flow. Hemisphere Pub Co, Mc- Graw Hill.

Pralits, J. O., L. Brandt and F. Giannetti, (2010). Instability and sensitivity of the flow around a rotating circular cylinder. Journal of Fluid Mechanics, 1-24.

Rashidi, S., M. Hayatdavoodi and J. A. Esfahani (2016). Vortex shedding suppression and wake control: a review. Ocean Engineering 129, $57-$ 80.

Sharma, A. and V. Eswaran (2004). Heat and fluid flow across a square cylinder in the twodimensional laminar flow regime. Numerical Heat Transfer 45, 247- 269.

Sheng, W. J., X. Y. Xin and T. Y. Sheng (2013). Active control of circular cylinder flow by affiliated rotating cylinders. Society of China Technology Science 56, 1187-1197.

Shimizu, Y. and Y. Tanida (1987). Fluid forces acting on cylinders of rectangular crosssection. Transaction Japan Society of Mechanical Engineering B 44, 2699-2706.
Shtendel, T. and A. Seifert (2014). Threedimensional aspects of cylinder drag reduction by suction and oscillatory blowing. International Journal of Heat Fluid Flows 45, 109-127.

Sohankar, A., C. Norberg and L. Davidson (1998). Low-Reynolds-number flow around a square cylinder at incidence: study of blockage, onset of vortex shedding and outlet boundary condition. International Journal of Numerical Methods in Fluids 26, 39-56.

Sohankar, A., M. Khodadian and E. Rangraz (2015). Control of fluid flow and heat transfer around a square cylinder by uniform suction and blowing at low Reynolds numbers. Computers and Fluids 109, 155-167.

Sumer, B. M. and J. Fredsoe (2006). Hydrodynamics around cylindrical structures, In revised (Ed.), Advanced series on ocean engineering 26. World Scientific Publishing Company.

Tropea, C., A. L. Yarin and J. F. Foss (2007). Springer handbook of experimental fluid mechanics, Printing and Binding, Sturtz $\mathrm{GmbH}$.

White, F.M. (2010). Fluid mechanics, Fourth ed., WCB McGraw-Hill.

Williamson, C. H. K. (1989). Oblique and parallel modes of vortex shedding in the wake of a circular cylinder at low Reynolds numbers. Journal of Fluid Mechanics 206, 579-627.

Zdravkovich, M. M. (1990). Conceptual overview of laminar and turbulent flows past smooth and rough circular cylinders .Journal of Wind Engineering and Industrial Aerodynamics 33, 53-62. 